\title{
The impact of the orientation on the lobe asymmetry in 3C328 radio galaxy
}

\author{
Andrzej Marecki and Mateusz Ogrodnik \\ Toruń Centre for Astronomy, Faculty of Physics, Astronomy, and Informatics, \\ Nicolaus Copernicus University, Toruń, Poland \\ email: amr@astro.uni.torun.pl
}

\begin{abstract}
In the double-lobed Fanaroff-Riley type-II radio galaxy 3C328, one lobe is devoid of a hotspot. We carried out the VLBA observations of the central component of 3C328 and we found there a jet pointing towards the lobe without a hotspot. We suggest that this is because the nucleus of $3 \mathrm{C} 328$ remained in off-state for some time in the recent past. No jets were produced during that period so both hotspots faded out. However, under the assumption that one lobe is much farther from the observer than the other, the hotspot of the far-side lobe can be perceived as not decayed yet due to the light-travel lag. Since activity of the nucleus has been restored, we obtain a proof that it is recurrent. It follows that the orientation of the jet is not a paradox.
\end{abstract}

Normally, both lobes of Fanaroff-Riley (FR) type-II radio galaxies are terminated with hotspots. In FR II radio galaxy 3C328, however, only one lobe has a hotspot whereas the other is clearly devoid of a hotspot (Fig.1). We carried out the Very Long Baseline Array (VLBA) observations of the central component of 3C328 and we found there a jet (Fig.2) oriented towards the southern lobe, i.e. the one with no hotspot.

At first sight, it is puzzling why, despite the presence of the jet seemingly fueling the southern lobe, it appears as a relic. We propose the following interpretation of this apparent paradox. Assume that the nucleus of 3C328 remained quiescent for some time in the recent past. Since no jets were produced in that period, the hotspots were not energized. As a result, they both faded out. To explain why they are asymmetric anyway, we assume is that the northern lobe is much farther from us than the southern one hence the hotspot of the former is perceived as not dispersed yet due to the light-travel lag. The jet was relaunched after a period of quiescence, it has therefore no causal connection with the relic it points at. Given that one-sided jets are always beamed towards the observer, the southern lobe must be on the near side in agreement with our second assumption.

This reasoning is similar to that used when explaining the Laing-Garrington effect (Laing 1988; Garrington et al. 1988), i.e. the correlation between the jet orientation and the degree of polarization of radio lobes. What we present here is an analogue of LaingGarrington effect but, instead of an asymmetry of polarization (that perhaps could also be present in 3C328), we observe an asymmetry of its lobes - the near-side lobe appears "older" than the far-side lobe. Such kind of asymmetry may emerge only because of the episodic nature of galactic activity. The images of $3 \mathrm{C} 328$ we perceive are thus a record of two subsequent transitions in its nucleus: from radio-loud to radio-quiet and back to radio-loud state. This way we obtain a proof that the activity of $3 \mathrm{C} 328$ must be recurrent.

\section{References}

Garrington, S. T., Leahy, J. P., Conway, R. G., \& Laing, R. A. 1988, Nature, 331, 147

Laing, R. A. 1988, Nature, 331, 149

Machalski, J., Condon, J. J. 1983, AJ, 88, 143 


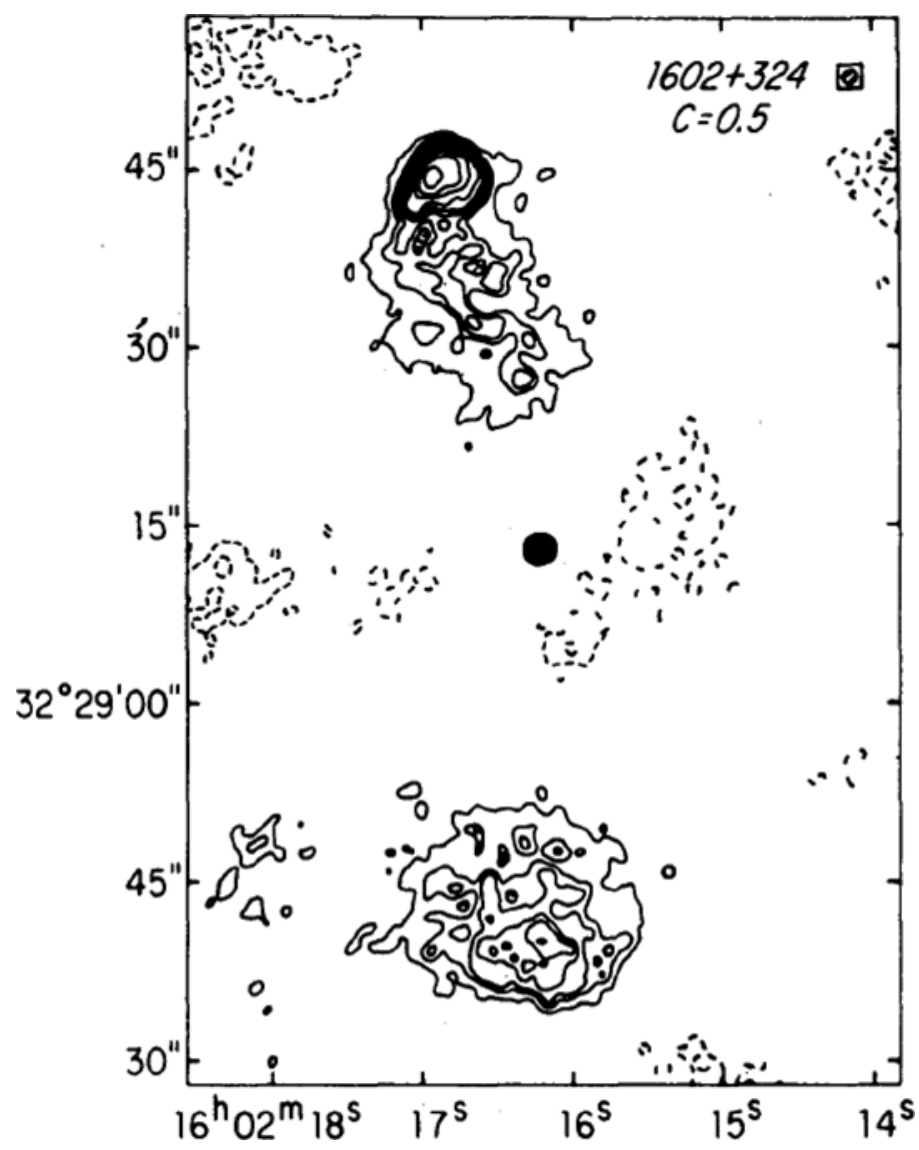

Figure 1. Very Large Array (VLA) image of 3C328 at $1465 \mathrm{MHz}$ (Machalski \& Condon 1983)

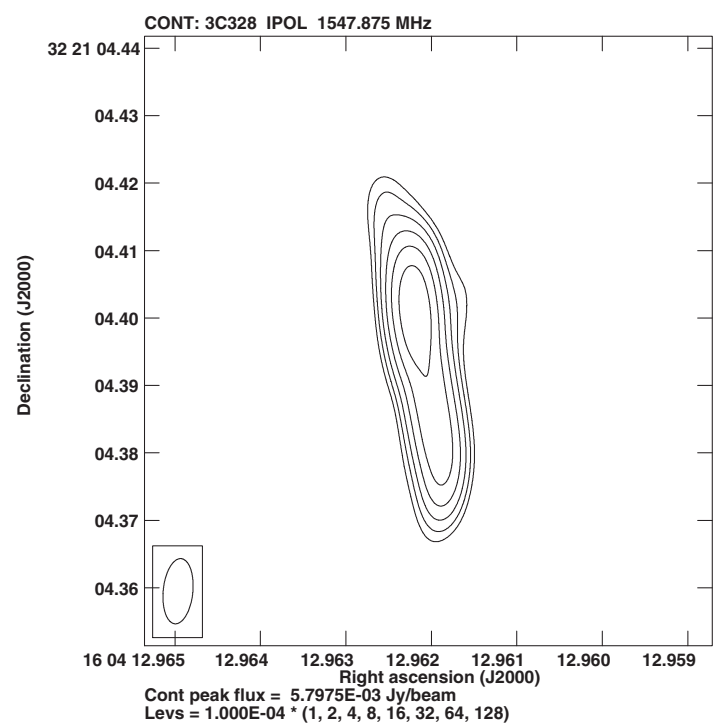

Figure 2. VLBA image of the central component of $3 \mathrm{C} 328$ at $1548 \mathrm{MHz}$ 